\title{
The structural feature surrounding glycated lysine residues in human hemo- globin
}

\author{
Shigenori Ito ${ }^{1}$, Takashi NAKAHARI ${ }^{2}$, and Daisuke YAMAмото ${ }^{3}$ \\ ${ }^{1}$ Department of Physiological Chemistry, ${ }^{2}$ Department of Physiology, and ${ }^{3}$ Biomedical Computation Center, Osaka Medical College, \\ 2-7 Daigakucho, Takatsuki 565-8686, Japan
}

(Received 24 March 2011; and accepted 13 April 2011)

\begin{abstract}
Complications derived from diabetes mellitus are caused by nonenzymatic protein glycation at the specific sites. LC/MS/MS was performed for the identification of the tryptic peptides of glycated hemoglobins using glyceraldehyde. After the identification of the glycation or non-glycation site, computer analysis of the structure surrounding the sites was carried out using PDB data (1BZ0). Five glycated lysine residues (Lys-16( $\alpha),-56(\alpha),-8(\beta),-82(\beta)$, and $-144(\beta))$ and four non-glycated lysine residues (Lys-7( $\alpha),-40(\alpha),-99(\alpha)$, and $-132(\beta))$ were identified. The non-glycated lysine residues, Lys-7 $(\alpha),-40(\alpha)$, and $-132(\beta)$, are most likely to form electrostatic interactions with the $\beta$ carboxyl group of Asp-74( $\alpha)$, C-terminal His-146( $\beta)$, and Glu-7( $\beta)$ by virtue of their proximity, which is $2.67-2.91 \AA(\mathrm{N}-\mathrm{O})$. Additionally, there are histidine residues within $4.55-7.38 \AA(\mathrm{N}-\mathrm{N})$ around eight sites except for Lys-7 $(\alpha)$. We conclude that the following factors seem to be necessary for glycation of lysine residues: (i) the apparent absence of aspartate or glutamate residues to inhibit the glycation reaction by forming an electrostatic interaction, (ii) the presence of histidine residues for acid-base catalysis of the Amadori rearrangement, and (iii) the presence of an amino acid residue capable of stabilizing a phosphate during proton transfer.
\end{abstract}

Complications derived from diabetes mellitus are caused by higher blood glucose level and consequent increases in nonenzymatic protein glycation known as the Maillard reaction. The first stage of this reaction involves the formation of Schiff's base, followed by the Amadori rearrangement. The second stage involves the formation of various complicated molecules, called advanced glycation end-products (AGEs). These molecules have long been thought to be the major cause of a series of complications, such as blindness, renal failure, stroke, and heart attack $(3,6,14,22)$. The initial reaction of the first stage pertains mainly to $\alpha$ and $\varepsilon$ amino groups of a protein in vivo. Most of the amino groups do not re-

Address correspondence to: Dr. Shigenori Ito Department of Physiological Chemistry, Osaka Medical College, 2-7 Daigakucho, Takatsuki 565-8686, Japan Tel: +81-72-683-1221(ext. 2968), Fax: +81-72-684-7032 E-mail: ito@art.osaka-med.ac.jp act with glucose, however, a limited number of them react with glucose. The reactivity is thought to depend on the structural environment around a glycation site. It is a method to control AGEs formation in order to prevent protein glycation. Glycation inhibitors, such as aminoguanidine and metformin, have been developed on the basis of this concept. If the structural characteristics of glycation sites can be defined more clearly, the development of various glycation inhibitors would be greatly accelerated.

Many glycated lysine residues on various proteins in vivo or in vitro have been identified by sequence analysis: human hemoglobin (17), human serum albumin (10), bovine pancreatic RNase A (23), and horse liver alcohol dehydrogenase (18). An acidic or lysine residue adjacent to a glycation site plays an important role in nonenzymatic glycation $(8,17)$. A histidine residue increases the rate of glycation of oligopeptides, whereas an acidic residue decreases the rate (5). A phosphate group is an effective cata- 
lyst of protein glycation (24). The Amadori rearrangement also requires a local acid-base catalysis (10). Thus, the glycation of horse liver alcohol dehydrogenase is catalyzed by an imidazole group and accelerated in the presence of phosphate (18). We have proposed that a phosphate ion would act as a mediator in the glycation process by accepting a proton from the catalytic histidine residue and donating it to the amino group, and that the formation between an amino group and an acidic group would bring about the impaired glycation (12). In this study, we inspected the structural features of lysyl glycation sites on the basis of the results of glycation experiments with human hemoglobin.

\section{MATERIALS AND METHODS}

Materials. D-Glucose was purchased from Wako Chemical Co (Osaka, Japan). Human hemoglobin $(\times 4$ crystallized) and glyceraldehyde were purchased from Nakarai Tesque Co. (Kyoto, Japan). TPCK (tosyl phenylalanyl chloromethyl ketone)-treated trypsin was obtained from Worthington Biochemicals Co. (Lakewood, USA). Desalting column PD-10 was purchased from Amersham Biosciences Co. (Piscataway, USA).

Glycation of human hemoglobin with glyceraldehydes. Human hemoglobin at a concentration $0.05 \mathrm{mM}$ of was incubated with $0.05 \mathrm{M}$ DL-glyceraldehyde in a phosphate-buffered saline (PBS, pH 7.4) at $37^{\circ} \mathrm{C}$ for 1.5 or $3.0 \mathrm{~h}$.

Preparation of globin by acid/acetone extraction. Fifty microliters of the hemoglobin PBS solution was added to $1.0 \mathrm{~mL}$ of cold acetone containing $1 \mathrm{~N} \mathrm{HCl}(50: 1, \mathrm{v} / \mathrm{v})$ in a $1.5-\mathrm{mL}$ microcentrifuge tube. The globin protein, isolated by centrifugation at 3,000 r.p.m. for $3 \mathrm{~min}$, was washed two times with $1.0 \mathrm{~mL}$ of cold acetone and was then washed with $1.0 \mathrm{~mL}$ of cold diethylether (20).

Peptide sequence analysis by LC/MS/MS. Globin dissolved in $0.1 \mathrm{M}$ ammonium bicarbonate was digested with TPCK-trypsin $(\mathrm{E} / \mathrm{S}=1 / 50)$ at $37^{\circ} \mathrm{C}$ for $5 \mathrm{~h}$. Liquid chromatography coupled with electrospray ionization tandem mass spectrometry (LC/MS/ MS) was employed for identification of the tryptic peptides. The HPLC system consisted of Waters 2690 Separation Module with a 2487 Dual 1 Absorbance Detector and a $2.1 \times 150 \mathrm{~mm}$ HPLC column (Waters X Terra MS C18, $5 \mathrm{~mm}$ ). A gradient elution was performed with eluent $\mathrm{A}(0.1 \%$ acetic acid and
$0.02 \%$ trifluoroacetic acid in water) and eluent $\mathrm{B}$ $(0.1 \%$ acetic acid and $0.02 \%$ trifluoroacetic acid in acetonitrile) at a flow rate of $0.2 \mathrm{~mL} / \mathrm{min}$ : $0 \mathrm{~min}$, $98 \% \mathrm{~A} ; 60 \mathrm{~min}, 60 \% \mathrm{~B}$. The eluate was monitored at $214 \mathrm{~nm}$ and then injected into a quadruple mass spectrometer with an electrospray ionized system (Finnigan LC Q DECA; Thermo Fisher Sci. Inc., Waltham, USA). For the sequence analyses of the mass spectra of tryptic peptides, an Xcalibur software system (Thermo Fisher Sci. Inc.) was used.

Computer analysis of glycation site. Analysis of the structure surrounding the glycation or non-glycation site was mainly carried out by distance measurements between the atoms in two target residues using Molecular Operating Environment (Chemical Computing Group Inc., Montreal, Canada, http:// www.chemcomp.com/). The structural dataset of human hemoglobin was obtained from the RCSB Protein Data Bank (http://www.rcsb.org/pdb/home/ home.do).

\section{RESULTS}

\section{Identification of the glycation sites on hemoglobin}

We identified five glycation sites and four nonglycation sites in human hemoglobin by peptide analysis. The tryptic peptides of native and two glycated (1.5 and $3.0 \mathrm{~h}$ incubation) hemoglobin proteins were purified by HPLC chromatography, as shown in Fig. 1A-C. Amino acid sequences of these peptides were determined by LC/MS/MS (Table 1). Fourteen and fifteen tryptic peptides are derived from $\alpha$ and $\beta$ chains of human hemoglobin, respectively (4). We recovered 6 ( $\alpha$ chain) and 8 ( $\beta$ chain) peptides: $\alpha$ chain; AT1 (peak number 2 , residue number 1-7), AT3 (1, 12-16), AT4 $(6,17-31)$, AT5 (13, 32-40), AT6 (12, 41-56), and AT11 (8, 93-99), and $\beta$ chain; BT1 (peak number 3 , residue number $1-8)$, BT4 (15, 31-40), BT9 (14, 67-82), BT10 (11, 83-95), BT11 (9, 96-104), BT12 (16, 105-120), BT13 (10, 121-132), and BT14 (7, 133-144) (Fig. 1 and Table 1). Glycated lysine residues are identified as an increase or decrease in relative peak height $(\mathrm{rPh})$ as compared to the peak marked with an asterisk (Fig. 1). This peak commonly emerged among three chromatograms (Fig. 1), had a constant height and no amino acid by MS/MS analysis. For example, the height of peak 1 (tryptic peptide AT3, residue $12-16$ of the $\alpha$ chain) reduces with time (Fig. 1). This peptide has a "slope value" of -0.58 , as calculated by the addition of two $\mathrm{rPh}$ values at a $1.5 \mathrm{~h}$ interval (Table 1) and indicates glycation of the lysine 
(A)

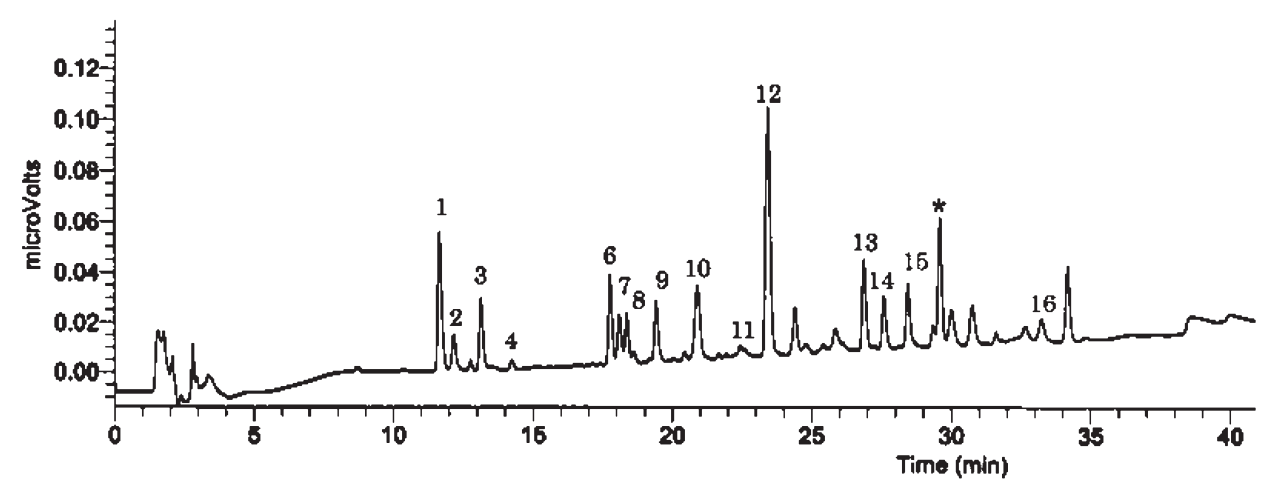

(B)

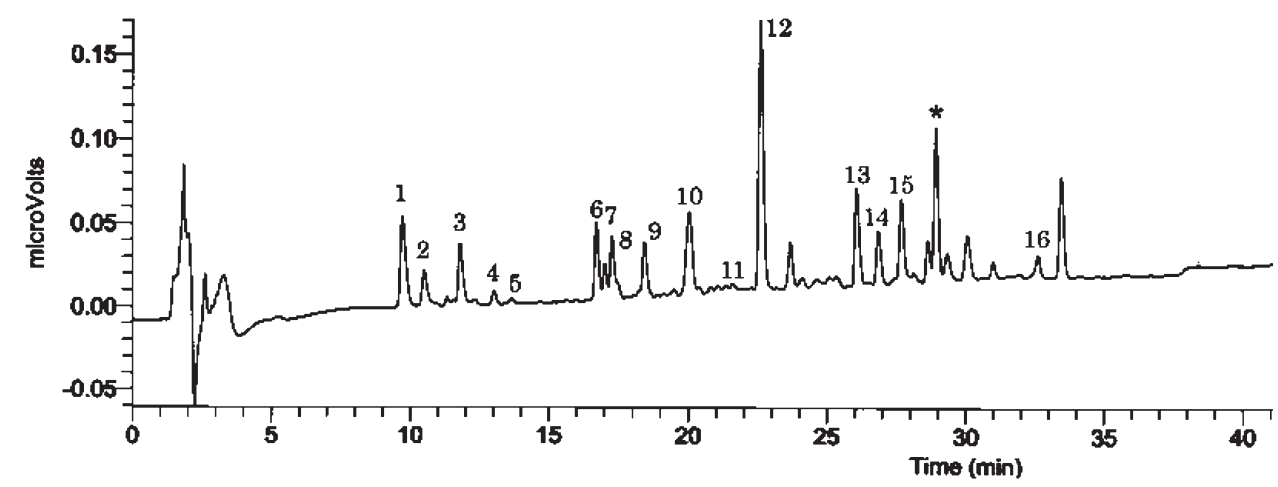

(C)

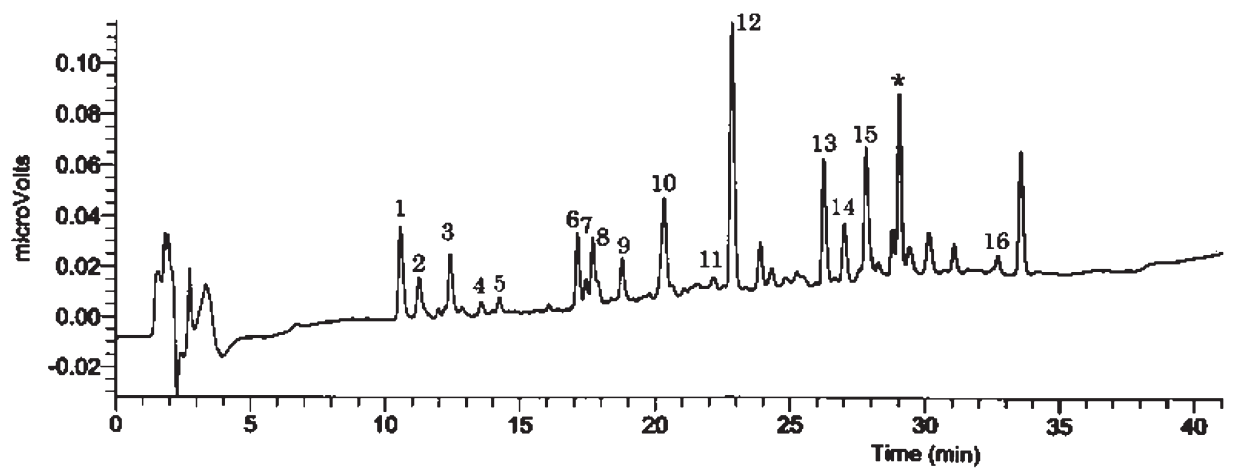

Fig. 1 HPLC chromatograms of the tryptic peptides of human hemoglobin. Samples for chromatography (A), (B), and (C) were the tryptic digests of hemoglobins incubated with DL-glycelaldehyde for $0,1.5$, and $3.0 \mathrm{~h}$, respectively. Peak numbers designate the identified tryptic peptides, as shown in Table 1. The relative peak heights were calculated on the basis of the peak marked by an asterisk.

residue. Glycation of the $\varepsilon$-amino group inhibits tryptic cleavage of the $\mathrm{C}$-terminal peptide bond at Lys-16( $\alpha)$. The fact that Lys-16 is labile to glycation is also supported by the parallel reduction of both peptide AT3 (peak 1) and AT4 (peak 6). Other glycation sites are identified using the same protocol that uses the "slope value". The yields of the fol- lowing peptides are reduced as glycation time is increased: AT3 (peak 1), AT6 (peak 12), BT1 (peak 3), BT9 (peak 14), BT11 (peak 9), and BT14 (peak 7) (Fig. 1 and Table 1). From these peptides, we identified five lysine glycation sites: Lys-16( $\alpha)$, Lys-56( $\alpha)$, Lys- $8(\beta)$, Lys-82( $\beta)$, and Lys-144( $\beta)$. Two peptides AT4 (peak 6) and BT11 (peak 9) have low values of 
Table 1 List of the tryptic peptides of human hemoglobin

\begin{tabular}{cclccc}
\hline Chain & Identifier & Amino acid sequence & Residue number & Peak no. & Slope value* \\
\hline$\alpha$ & AT1 & VLSPADK & $1-7$ & 2 & -0.06 \\
& AT2 & & $8-11$ & N.D. & \\
& AT3 & AAWGK & $12-16$ & 1 & -0.58 \\
& AT4 & VGAHAGEYGAEALER & $17-31$ & 6 & -0.32 \\
& AT5 & MFLSFPTTK & $32-40$ & 13 & -0.03 \\
& AT6 & TYFPHFDLSHGSAQVK & $41-56$ & 12 & -0.47 \\
AT7-10 & & $57-92$ & N.D. & \\
AT11 & VDPVNFK & $93-99$ & 8 & 0.00 \\
& AT12-14 & & $100-141$ & N.D. & \\
BT1 & VHLTPEEK & $1-8$ & 3 & -0.23 \\
& BT2-3 & & $9-30$ & N.D. & \\
BT4 & LLVVYPWTQR & $31-40$ & 15 & 0.21 \\
BT5-8 & & $41-66$ & N.D. & \\
BT9 & VLGAFSDGLAHLDNLK & $67-82$ & 14 & -0.10 \\
BT10 & GTFATLSELHCDK & $83-95$ & 11 & -0.02 \\
BT11 & LHVDPENFR & $96-104$ & 9 & -0.26 \\
BT12 & LLGNVLVCVLAHHFGK & $105-120$ & 16 & -0.01 \\
BT13 & EFTPPVAAYQK & $121-132$ & 10 & -0.05 \\
BT14 & VVAGVANALAHK & $133-144$ & 7 & -0.23 \\
BT15 & & $145-146$ & N.D. & \\
\hline
\end{tabular}

* This value is calculated by addition of two relative-peak-height values, which decrease at each $1.5 \mathrm{~h}$ interval. N.D.: Not Detected

-0.32 and -0.26 , respectively (Table 1 ). Hydroxypyruvaldehyde is produced from glyceraldehyde autoxidation in phosphate buffer $(\mathrm{pH} 7.4)$ at $37^{\circ} \mathrm{C}(15)$. This by-product may react with arginine residues in hemoglobin (16). Accordingly, we did not include the Arg-31( $\alpha)$ and Arg-104( $\beta)$ residues in our analyses. It is thought that Lys-7( $\alpha)$, Lys-40( $\alpha)$, Lys-99( $\alpha)$, and Lys-132( $\beta)$ are non-glycation sites because of the small "slope value" of AT1 (peak 2), AT5 (peak 13), AT11 (peak 8), and BT13 (peak 10) (Table 1). Peptide BT10 (peak 11) and BT12 (peak 16) were not analyzed because of their low yields.

Analysis of the structure of the glycation site

The structural environments surrounding nine lysine residues Lys-7 $(\alpha)$, Lys-16( $\alpha)$, Lys-40( $\alpha)$, Lys-56( $\alpha)$, Lys-99( $\alpha)$, Lys-8( $\beta)$, Lys- $82(\beta)$, Lys-132( $\beta)$ and Lys$144(\beta)$ were analyzed by MOE program using PDB data (1BZ0). We investigated the structure according to the answers to the following five questions that were based on the results from our previous study (12): (i) Where is the $\varepsilon$-amino group on the hemoglobin molecule? (ii) Is there an acidic residue adjacent to the site capable of forming an electrostatic interaction with a protonated amino group? (iii) Is there a histidine residue close to the glycation site that can act as a catalyst for the Amadori rearrangement? (iv) Is there a stabilizing residue capable of forming a phosphate bridge between glycation and catalytic sites? And finally, (v) the distances between the $\mathrm{N}$ atom of the $\varepsilon$-amino group, the $\mathrm{N}$ atom of the histidine residue, and any atom of a stabilizing residue are measured in the modeling. The resulting data are summarized in Table 2. All sites are located on the surface of the hemoglobin molecule, except for the non-glycated Lys-99( $\alpha)$ site. Molecular models of the structure around Lys-16( $\alpha)$, Lys$40(\alpha)$, and Lys-99( $\alpha)$ are shown in Fig. 2. The nonglycated lysine residues Lys-7( $\alpha)$, Lys-40 $(\alpha)$, and Lys-132( $\beta)$ are most likely to form electrostatic interactions with the $\beta$ carboxyl group of Asp-74( $\alpha)$, the C-terminal carboxyl group of His-146( $\beta)$ and the $\gamma$ carboxyl group of Glu-7( $\beta)$ by virtue of their proximity, 2.67-2.91 $\AA$ ( $\mathrm{N}-\mathrm{O})$ (Table 2$)$. There are histidine residues within $4.55-7.38 \AA(\mathrm{N}-\mathrm{N})$ around eight sites except for Lys-7 $(\alpha)$. However, the nonglycated Lys-99( $\alpha)$, which cannot form any electrostatic interactions, was located in a concealed area, as shown in Fig. 2C.

\section{DISCUSSION}

In our experiments, we employed glyceraldehyde instead of glucose for protein glycation. A nonenzymatic glycation reaction between the aldehyde group of glucose and the amino group of hemoglobin fol- 
Table 2 Distances between glycation site, catalytic site, and stabilizing site

\begin{tabular}{|c|c|c|c|c|c|c|}
\hline \multirow{2}{*}{$\begin{array}{l}\text { Glycation }(\mathrm{G}) \text { or } \\
\text { non-glycation }(\mathrm{N})\end{array}$} & \multirow{2}{*}{$\begin{array}{l}\text { Residue number } \\
\text { (chain) }\end{array}$} & \multirow{2}{*}{ Distribution } & Catalytic residue & \multicolumn{2}{|l|}{ Stabilizing residue } & \multirow{2}{*}{$\begin{array}{l}\text { Acidic residue } \\
\text { Distance }(\AA)^{* 1}\end{array}$} \\
\hline & & & Distance $(\AA)^{* 1}$ & Distance $(\AA)^{* 1}$ & Distance $(\AA)^{* 2}$ & \\
\hline \multirow[t]{2}{*}{$\mathrm{N}$} & Lys-7( $\alpha)$ & surface & N.D. & N.D. & & Asp-74( $\alpha)$ \\
\hline & & & & & & $2.67(\mathrm{OD} 1)$ \\
\hline \multirow[t]{2}{*}{ G } & Lys-16( $\alpha)$ & surface & His- $20(\alpha)$ & Lys-16( $\alpha)$ & & Glu- $166(\alpha)$ \\
\hline & & & 7.38(NE2) & $4.95(\mathrm{~N})$ & 5.86 & $4.22(\mathrm{OE} 1)$ \\
\hline \multirow[t]{2}{*}{$\mathrm{N}$} & Lys-40( $\alpha)$ & surface & His- $146(\beta)$ & Cys-93( $\beta)$ & & His- $146(\beta)$ \\
\hline & & & $5.19(\mathrm{ND} 1)$ & $7.45(\mathrm{SG})$ & 5.43 & $2.69(\mathrm{O})$ \\
\hline \multirow[t]{2}{*}{ G } & Lys- $56(\alpha)$ & surface & His- $50(\alpha)$ & His- $50(\alpha)$ & & N.D. \\
\hline & & & 7.73(NE2) & $6.95(\mathrm{O})$ & 4.01 & \\
\hline \multirow[t]{2}{*}{$\mathrm{N}$} & Lys-99( $\alpha)$ & cavity & His- $103(\alpha)$ & Lys-99( $\alpha)$ & & N.D. \\
\hline & & & $5.82(\mathrm{ND} 1)$ & $4.14(\mathrm{O})$ & 5.92 & \\
\hline \multirow[t]{2}{*}{ G } & Lys- $8(\beta)$ & surface & His- $2(\beta)$ & Val-1 $(\beta)$ & & Asp-79( $\beta)$ \\
\hline & & & 7.34(NE2) & $6.89(\mathrm{O})$ & 5.07 & $4.40(\mathrm{OE} 1)$ \\
\hline \multirow[t]{2}{*}{ G } & Lys- $82(\beta)$ & surface & His- $143(\beta)$ & Asn-139( $\beta)$ & & N.D. \\
\hline & & & $5.18(\mathrm{NE} 2)$ & $6.77(\mathrm{O})$ & 6.45 & \\
\hline \multirow[t]{2}{*}{$\mathrm{N}$} & Lys-132( $\beta)$ & surface & His-2( $\beta)$ & His- $2(\beta)$ & & Glu-7( $\beta)$ \\
\hline & & & $4.55(\mathrm{ND} 1)$ & $3.60(\mathrm{O})$ & 2.89 & $2.91(\mathrm{OE} 1)$ \\
\hline \multirow[t]{2}{*}{ G } & Lys- $144(\beta)$ & surface & His- $143(\beta)$ & Lys-144( $\beta)$ & & Glu- $90(\beta)$ \\
\hline & & & $5.24(\mathrm{ND} 1)$ & $6.46(\mathrm{O})$ & 7.17 & $4.00(\mathrm{OE} 1)$ \\
\hline
\end{tabular}

${ }^{* 1}$ The numbers show the distance $(\AA)$ from $\mathrm{N}$ atom of glycation site, and the symbol in parentheses shows the atom position in the amino acid residue.

${ }^{* 2}$ The number shows the distance $(\AA)$ between the atoms of catalytic and phosphate-stabilizing residues.

lows these two steps: Schiff's base formation and the Amadori rearrangement, an intramolecular reduction. Glucose forms glucopyranose in water via an intramolecular interaction between an aldehyde group and a hydroxyl group, and the free aldehyde group that takes part in the formation of the Schiff's base is lost (7). Glyceraldehyde, which does not form a ring in water, has been employed as an alternative to glucose to obtain glycation products quickly $(1,2)$. In addition, it is difficult to determine the glycation sites in the case of glucose because of various modifications by complicated by-products derived from lengthy incubation with glucose (21).

We evaluated the structures of glycated or nonglycated sites in human hemoglobin by molecular modeling. It has been pointed out that the limited number of glycation sites indicates some type of selectivity in the reaction of sugar with hemoglobin (1). The formation of glycation sites in hemoglobin molecules is a consequence of its three-dimensional structure around these sites (15). The Amadori rearrangement, by which a reducing sugar is fixed on a protein, is accelerated with an acid-base catalyst (9, $10,11,13)$. An imidazole group of a histidine residue is most likely to play a significant role in this reaction. A histidine residue in the vicinity of Val$1(\beta)$ of hemoglobin is thought to be a catalyst (5).
The imidazole group can, however, work as a catalyst in the presence of an intermediary to transfer a proton. It is known that phosphate enhances the rate of glycation in some proteins (24) and oligopeptides (5). We have predicted, by computer analysis of the structure environment surrounding the $\beta$-N-terminal glycation site of a hemoglobin molecule, that the glycation site would have the following two characteristics: (i) glycation of an amino group is probably accelerated by the neighboring histidine residue, which acts as an acid-base catalyst via phosphatemediated proton transfer; and (ii) the formation of an electrostatic interaction between an amino group and a neighboring carboxyl group would bring about impaired glycation of the amino group (12). In order for the histidine residues to play the role of catalyst, it may be necessary to stabilize the phosphate group with three points: the target site (Schiff's base), a catalytic site (imidazole group), and one other stabilizing site, thus making the so-called "phosphate triangle" (12). The dimensions of the triangle in a phosphate binding protein (PDB; $2 \mathrm{ABH}$ ) range from 2.29 to $7.42 \AA$. Five glycation sites satisfy these requirements (Table 2 ). However, Lys$40(\alpha)$ and Lys-132( $\beta)$ are identified as non-glycation sites in spite of satisfying these requirements. We suggest that the positively-charged $\varepsilon$ amino groups 
(A)

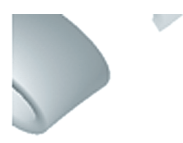

(B)
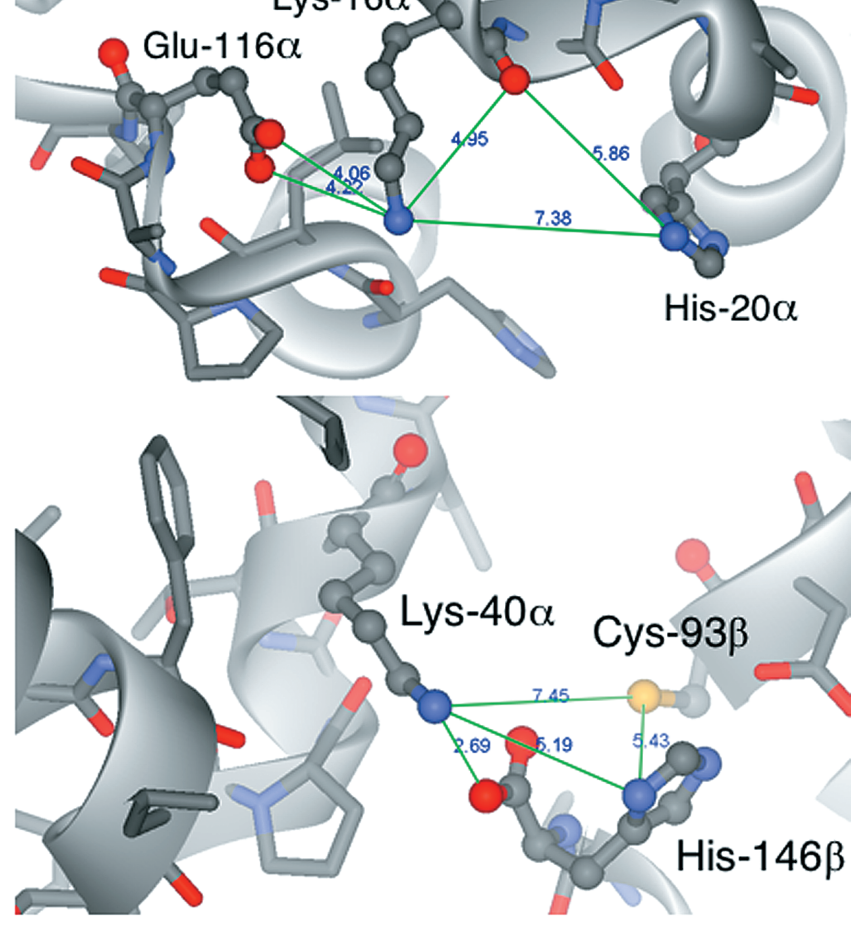

Fig. 2 Structure of glycated or non-glycated sites on human hemoglobin molecules (PDB data; 1BZ0). (A) Glycation site; Lys-16(a). (B) Non-glycation site; Lys-40(a). Nitrogen, oxygen, and carbon atoms are shown in blue, red, and grey, respectively. The line indicates the interaction between an amino and a carboxyl group, and the triangle indicates the "phosphate triangle," which comprises the $\mathrm{N}$ atom of a lysine residue, $\mathrm{N}$ atom of a histidine residue, and a stabilizing atom of a phosphate group. Each figure shows the distance $(\AA)$ between atoms. There is no interaction between Glu-116a and Val-1a. Lys-40a interacted with His-146 $\beta$. (C) Non-glycation site; Lys-99(a).

(C)

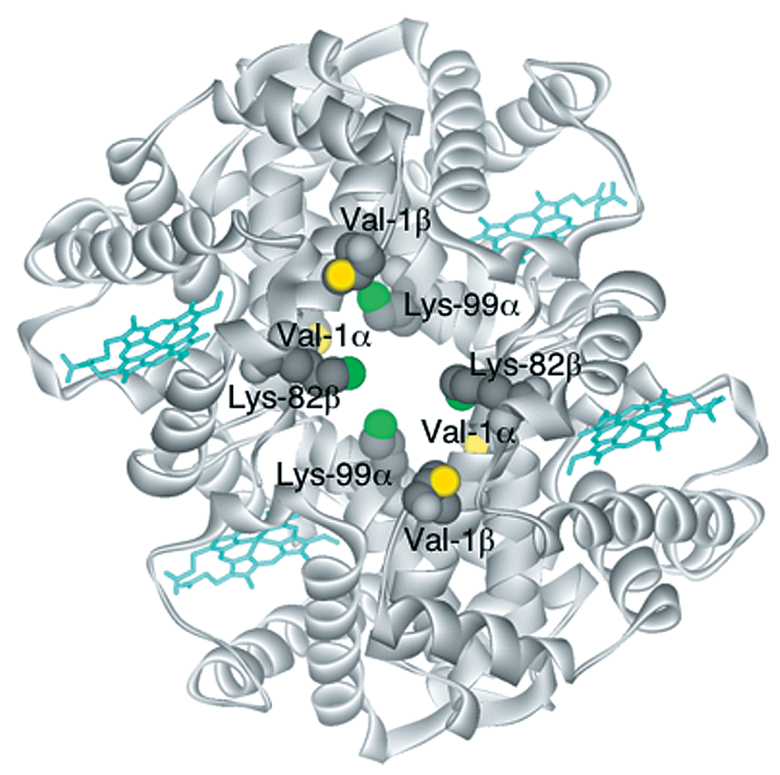

of these residues would form stable electrostatic interactions with the neighboring negatively-charged carboxyl groups of Glu-7( $\beta)$ and terminal His-146( $\beta)$, respectively. A protonated $\mathrm{N}$ atom of an amino group cannot attack a carbonyl carbon. A carboxyl group near a glycation site leads the glycation rate to decrease (5). Furthermore, it was reported that glycation would be inhibited by an adjacent carboxyl group with an electrostatic interaction (13). Nonglycated Lys- $7(\alpha)$ is expected to form an electrostatic interaction with the $\beta$ carboxyl group of Asp-74( $\alpha)$ without forming the "phosphate triangle" (Table 2). Lys-99( $\alpha)$ has a "phosphate triangle" but no neighboring acidic residue is recognized as a non-glyca- 
tion site (Table 2). We believe that a phosphate is unable to access the site because it is located in a cavity surrounded by four chains of hemoglobin (Fig. 2C).

Next to collagen, which has a low metabolic turnover, hemoglobin $(\mathrm{Hb})$ would contribute to the increase in the amount of AGEs at higher blood glucose levels (22). Glycation of lysine residues on $\mathrm{Hb}$ molecules would contribute to AGEs formation, besides that of the N-terminal valine of $\mathrm{HbAlc}$, which is well known to be a diagnostic tool for diabetes (19). The reactions for AGEs formation proceed after the attack of a nucleophilic reagent on the carbonyl group of Amadori products. Just like the Amadori rearrangement, the rate of these reactions will be accelerated by an acid-base catalyst. Therefore, we think that the findings of this study will be very useful for elucidating of the reaction mechanism of AGEs production.

In conclusion, the following features of the structure surrounding glycated lysine residues in human hemoglobin are thought to be important: (i) the absence of aspartate or glutamate residues to inhibit the glycation reaction by forming an electrostatic interaction, (ii) the presence of a histidine residue for acid-base catalysis of the Amadori rearrangement, and (iii) the presence of an amino acid residue able to stabilize a phosphate for proton transfer.

\section{Acknowledgements}

The authors thank Assistant Professor Ayako Miyazaki for her friendly technical support in the LC/ MS/MS analysis.

\section{REFERENCES}

1. Acharya AS and Manning JM (1980) Reactivity of the amino groups of carbonomonoxyhemoglobin $\mathrm{S}$ with glyceraldehydes. J Biol Chem 255, 1406-1412.

2. Acharya AS, Sussman LG and Manning JM (1983) Schiff's base adducts of glyceraldehydes with hemoglobin. $J$ Biol Chem 258, 2296-2302.

3. Ahmed N (2005) Advanced glycation endproducts - role in pathology of diabetic complications. Diabetes Res Clin Pract 67, 3-21.

4. Amino acid sequence data of globins (1972) In: Atlas of Protein Sequence and Structure Vol. 5 (Dayhoff MO, ed.), Silver Spring: National Biomedical Research Foundation, D-56 and D-64.

5. Bai Y, Ueno H and Manning JM (1989) Some factors that influence the nonenzymatic glycation of peptides and polypeptides by glyceraldehydes. J Protein Chem 8, 299-315.
6. Brownlee M, Vlassara MH and Cerami A (1984) Nonenzymatic glycosylation and the pathogenesis of diabetic complications. Ann Internal Med 101, 527-537.

7. Bunn HF and Higgins PJ (1981) Reaction of monosaccharides with proteins: Possible evolutionary significance. Science 213, 222-224.

8. Garlick RL and Mazer JS (1983) The principal site of nonenzymatic glycosylation of human serum albumin in vivo. $J$ Biol Chem 258, 6142-6146.

9. Hodge JE (1955) The Amadori rearrangement. Adv Carbohydr Chem 10, 169-203.

10. Iberg N and Flückiger R (1986) Nonenzymatic glycosylation of albumin in vivo. Identification of multiple glycosylated sites. J Biol Chem 261, 13542-13545.

11. Isbell HS and Frush HL (1958) Mutarotation, hydrolysis, and rearrangement reactions of glycosylamines. J Org Chem 23, 1309-1319.

12. Ito S, Nakahari T and Yamamoto D (2010) Relationship between impaired glycation and the N-terminal structure of the $\mathrm{Hb}$ Görwihl [ $\beta 5$ (A2)Pro $\rightarrow$ Ala] variant. Hemoglobin 34, 1-6.

13. Lowrey CH, Lyness SJ and Soeldner JS (1985) The effect of hemoglobin ligands on the kinetics of human hemoglobin A1c formation. $J$ Biol Chem 260, 11611-11618.

14. McCance DR, Dyer DG, Dunn JA, Bailie KE, Thorpe SR, Baynes JW and Lyons TJ (1993) Maillard reaction products and their relation to complications in insulin-dependent diabetes mellitus. J Clin Invest 91, 2470-2478.

15. Nacharaju P and Acharya AS (1992) Amadori rearrangement potential of hemoglobin at its glycation sites is dependent on the three-dimensional structure of protein. Biochemistry 31, 12673-12679.

16. Nakaya K, Horinishi H and Shibata K (1967) States of amino acid residues in proteins XIV, Glyoxal as a reagent for discrimination of arginine residues. J Biochem 61, 345-351.

17. Shapiro R, McManus MJ, Zalut C and Bunn HF (1980) Sites of nonenzymatic glycosylation of human hemoglobin A. $J$ Biol Chem 255, 3120-3127.

18. Shilton BH, Campbell RL and Walton DJ (1993) Site specificity of glycation of horse liver alcohol dehydrogenase in vitro. Eur J Biochem 215, 567-572.

19. Steffes M, Cleary P, Goldstein D, Little R, Wiedmeyer HM, Rohlfing C, England J, Bucksa J, Nowicki M and DCCT/ EDIC Research Group (2005) Hemoglobin A1c measurements over nearly two decades: Sustaining comparable values throughout the diabetes control and complications trial and the epidemiology of diabetes interventions and complications study. Clin Chem 51, 753-758.

20. Teale FW (1959) Cleavage of the haem-protein link by acid methylethylketone. Biochim Biophys Acta 35, 543.

21. Thornalley PJ (1985) Monosaccharide autoxidation in health and disease. Environ Health Pers 64, 297-307.

22. Ulrich P and Cerami A (2001) Protein glycation, diabetes, and aging. Recent Prog Horm Res 56, 1-22.

23. Watkins NG, Thorpe SR and Baynes JW (1985) Glycation of amino groups in protein. Studies on the specificity of modification of RNase by glucose. J Biol Chem 260, 10629-10636.

24. Watkins NG, Neglia-Fisher CI, Dyer DG, Thorpe SR and Baynes JW (1987) Effect of phosphate on the kinetics and specificity of glycation of protein. J Biol Chem 262, 72077212 . 